\title{
On hereditary models of polymers
}

\author{
Monica De Angelis \\ Università Studi di Napoli Federico II, Facoltà di Ingegneria, \\ Dip. Mat. e Appl., via Claudio 21, 80125, Napoli; Italy. \\ Tel. 0817683387 e-mail modeange@unina.it
}

\begin{abstract}
An equivalence between an integro-

differential operator $\mathcal{M}$ and an evolution operator $\mathcal{L}_{n}$ is determined. Owing to this equivalence, the fundamental solution of $\mathcal{L}_{n}$ is estimated in terms of the fundamental solution related to the third order operator $\mathcal{L}_{1}$ whose behaviour is now acquired. Moreover, properties typical of wave hierarchies can be applied to polymeric materials. As an example the case $n=2$ is considered and results are applied to the Rouse model and the reptation model which describe different aspects of polymer chains.
\end{abstract}

$$
g_{n}(t)=\sum_{h=1}^{n} B_{h} e^{-\beta_{h} t}
$$

where $n, B_{h}$ and $\beta_{h}$ depend on the polymer physics and are determined in order to fit the experimental curves for $g_{n}(t)$ with a required approximation [1-3].

Moreover, according to the well-known Muntz and Schwartz'z theorem [4-5], every continuous spectrum can be uniformly represented by Dirichlet polynomials.

In this paper, an integro - differential operator $\mathcal{M}$ related to (1.1) is considered and a conditioned equivalence between $\mathcal{M}$ and the $(2+n)$ order operator:

$$
\mathcal{L}_{n}=\partial_{t}^{(n)}\left(\partial_{t t}-c_{n}^{2} \partial_{x x}\right)+
$$

\section{Introduction}

The creep and relaxation processes related to the viscoelastic behavior of many actual polymeric materials are specified by means of memory functions like:

$$
a_{n-1} \partial_{t}^{(n-1)}\left(\partial_{t t}-c_{n-1}^{2} \partial_{x x}\right)+. . a_{0}\left(\partial_{t t}-c_{0}^{2} \partial_{x x}\right)
$$

where $a_{k}(k=0 . . n-1)$ are positive constants, is extablished.

By this equivalence whatever $n$ may be the fundamental solution of (1.2) is 
explictly determined and an estimate in terms of the fundamental solution related to the third order operator is achieved, too.

As applications, when $n=2$, results are applied to polymer chains and the Rouse model and the reptation model are considered.

\section{Statement of the prob- lem}

Let $\mathcal{B}$ a linear, isotropic, homogeneous system and let $\underline{u}(x, t) \underline{i}$ the displacement field from an underformed reference configuration $\mathcal{B}_{0}$.

Indicating by $\sigma$ and $\varepsilon$ the only non vanishing components of the stress and the strain tensors, the constitutive relation is

$$
\sum_{k=0}^{n} a_{k} \partial_{t}^{k} \sigma=\sum_{k=0}^{n} \alpha_{k} \partial_{t}^{k} \varepsilon
$$

with $a_{k}, \alpha_{k}$ constant $\left(a_{n}, \alpha_{n} \neq 0\right)$.

So, if $\underline{\mathbf{f}}=f \underline{\mathbf{i}}$ is the known body force, $\rho_{0}$ denotes the mass density in $\mathcal{B}_{0}$, and $c_{k}=\alpha_{k} / \rho_{0} a_{k}$, the one dimensional linear motions of $\mathcal{B}$ are described by higher order equation $[6]$ :

$$
\mathcal{L}_{n} u=F
$$

where

$$
\mathcal{L}_{n}=\sum_{k=0}^{n} a_{k} \partial_{t}^{(k)}\left(\partial_{t t}-c_{k} \partial_{x x}\right)
$$

and

$$
F=\left(1 / \rho_{0}\right) \sum_{k=0}^{n} a_{k} \partial_{t}^{k} f
$$

In (2.3) constants $c_{k}$ are the characterized speeds depending on the materials properties of the medium and in many physical problems it results $c_{0}^{2}<c_{1}^{2} . .<$ $c_{n-1}^{2}<c_{n}^{2}$ and so the equation (1.2) is typical of the wave hierarchies. [7].

When $n=1,(2.3)$ turns into a strictly hyperbolic third - order operator which models the evolution of the Standard Linear Solid (S.L.S.) [8-9] and its behaviour has been already discussed in [10]. The fundamental solution $\mathcal{E}_{1}$ has been explicitly determined together with maximum theorems and boundary layer estimates.

Moreover, if $J(t)$ denotes the creepcompliance, the behavior of most viscoelastic media is fairly well modelled by linear hereditary equations like:

$$
\varepsilon(t)=J(0) \quad[\sigma(t)+
$$

$$
\left.+\int_{-\infty}^{t} \dot{J}(t-\tau) \sigma(\tau) d \tau\right]
$$

According to fading memory hypotheses [11-12], $\dot{J}(t)$ is a positive fast decreasing function and in many real materials as polymers rubbers or bitumines, it is representated by means of chains of S.L.S. elements in series or parallel [2-3]. In the series case, one has:

$$
J_{n}(t)=J_{n}(0) \quad[1+
$$




$$
\left.+\sum_{k=1}^{n} \frac{B_{k}}{\beta_{k}}\left(1-e^{-\beta_{k} t}\right)\right]
$$

where $n$ is the number of elements in the chain, $J_{n}(0)$ denotes the elastic compliances and $\tau_{k}=\beta_{k}^{-1}$ are the characteristic times .

Let consider equations of one dimensional motions of $\mathcal{B}$ :

$$
\rho_{0} u_{t t}=\sigma+f, \quad \varepsilon=u,
$$

by means of $(2.5)$ - (2.7) the following integral constitutive equation is deduced:

$$
\begin{gathered}
\mathcal{M} u=c^{2} u_{x x}-u_{t t}- \\
-\int_{0}^{t} g(t-\tau) u_{\tau \tau} d \tau=-F_{*}(x, t)
\end{gathered}
$$

where

$$
c^{2}=\left[\rho_{0} J_{n}(0)\right]^{-1},
$$

$F_{*}=c^{2}\left[J_{n}(0) f+\int_{-\infty}^{0} \dot{J}_{n}(t-\tau) \sigma_{x}(\tau) d \tau\right]$.

and

$g=g_{n}(t)=\sum_{k=1}^{n} B_{k} e^{-\beta_{k} t}=\dot{J}_{n}(t) / J_{n}(0)$.

In this memory function, $n$ is quite arbitrary and constants $B_{k}$ and frequencies $\beta_{k}$ are such that:

$$
0<\beta_{1}<\beta_{2} \ldots<\beta_{n}
$$

$$
B_{k}>0 \quad \forall k=1,2 \ldots n .
$$

Whatever $\mathrm{n}$ may be, the fundamental solution $E_{n}$ of operator $\mathcal{M}$ has been explictly determined [13-14]. Moreover, let $E_{1}$ the fundamental solution related to an appropriate S.L.S. $\mathcal{B}_{1}^{*}$ defined by:

$$
g_{1}=b e^{-\beta_{1} t}
$$

with

$$
b=\beta_{1} \sum_{1}^{n} \frac{B_{k}}{\beta_{k}}
$$

the following theorem assures that the fundamental solution $E_{n}$ can be rigorously estimated by means of $E_{1}$.

In fact, if $\Gamma$ is the open forward characteristic cone $\{(t, x): t>0|x|<c t\}$, and $\chi_{n}=\prod_{k=2}^{n}\left(\frac{B_{k}}{\beta_{1}}\right)^{2}$, then the following theorem holds:

Theorem 1.1 - If the memory function is given by (2.10) (2.11), then the fundamental solution $E_{n}$ of $\mathcal{M}$ is a never negative $C^{\infty}(\Gamma)$ function and it satisfies the estimate:

$$
\begin{gathered}
0<E_{n}\left(\beta_{1} . . \beta_{n}, B_{1} . . B_{n}\right)< \\
<\chi_{n} \quad E_{1}\left(\beta_{1}, b\right)
\end{gathered}
$$

everywhere in the cone $\Gamma$ and whatever $n$ may be. 
3. Conditioned equivalence be- So, for $k=0, \ldots n-1$, one has: tween operators $\mathcal{L}_{n}$ and $\mathcal{M}$

$$
0<c_{k}<c_{n}=c^{2} .
$$

Let be null the initial data related to (2.2) and (2.8) and let

$$
\begin{gathered}
\mu_{k}=a_{k} / a_{n}, \\
\lambda_{k}=a_{k} c_{k} / a_{n} c_{n} \quad(k=0, . . n) .
\end{gathered}
$$

$$
0<c_{0}<c_{1} \ldots<c_{n} .
$$

Appling the Laplace transform and the polinomial identity one has $c_{n}=c^{2}$ and:

$(3.2)\left\{\begin{array}{l}\lambda_{0}=\beta_{1} \beta_{2} . . \beta_{n} \\ \ldots \ldots \ldots \ldots \ldots \ldots \ldots \ldots \ldots \\ \lambda_{n-2}=\beta_{1} \beta_{2}+\beta_{1} \beta_{3}+. . \beta_{n-1} \beta_{n} \\ \lambda_{n-1}=\beta_{1}+\ldots .+\beta_{n}\end{array}\right.$

So, owing to (2.11), all the $\lambda_{k}$ 's are positive. Further, as for $\mu_{k}$, one has:

As consequence, the following property holds:

Property 3.1 Hypotheses of fading memory (2.10) (2.11) imply that the differential operator (2.3) is typical of wave hierarchies.

Vice versa when the differential equation (2.2) is prefixed, to obtain the dual hereditary equation (2.8) with a memory function $g_{n}(t)$ satisfying $(2.10),(2.11)$, appropriate restrictiones on the constants $a_{k}, c_{k}$ must be imposed.

\section{Example 3.1}

When $n=2$, one has $c^{2}=c_{2}, B_{0}=1$. Further $\beta_{1}, \beta_{2}$ are real iff:

$$
\left\{\begin{array}{c}
\mu_{0}=\lambda_{0}+B_{1}\left(\beta_{2} . . \beta_{n}\right)+\ldots \\
\ldots+B_{n}\left(\beta_{1} . . \beta_{n-1}\right) \\
\ldots \ldots \ldots \ldots \ldots \ldots \ldots \ldots \\
\mu_{n-2}=\lambda_{n-2}+B_{1}\left(\beta_{2}+. .+\beta_{n}\right) \\
\ldots+B_{n}\left(\beta_{1}+. .+\beta_{n-1}\right) \\
\mu_{n-1}=\lambda_{n-1}+B_{1}+\ldots .+B_{n}
\end{array}\right.
$$

and (2.11), (3.3) imply too:

$$
\begin{aligned}
& \beta_{1}=\frac{1}{2 a_{2} c_{2}}\left(a_{1} c_{1}-\omega\right), \\
& \beta_{2}=\frac{1}{2 a_{2} c_{2}}\left(a_{1} c_{1}+\omega\right),
\end{aligned}
$$


it results $0<\beta_{1}<\beta_{2}$.

Moreover as for $B_{i}(i=1,2)$, one has:

$$
\begin{aligned}
& B_{i}=\frac{(-1)^{i-1}}{\omega}\left[a_{0}\left(c_{2}-c_{0}\right)-\right. \\
& \left.-a_{1} \beta_{i}\left(c_{2}-c_{1}\right)\right], \quad(i=1,2),
\end{aligned}
$$

and hence, $B_{1}>0, B_{2}>0$ iff

$$
\beta_{1}<\frac{a_{o}}{a_{1}} \frac{c_{2}-c_{0}}{c_{2}-c_{1}}<\beta_{2} .
$$

Therefore, the fourth-order operator:

$$
a_{2}\left(u_{t t}-c_{2} u_{x x}\right)_{t t}+
$$

$$
+a_{1}\left(u_{t t}-c_{1} u_{x x}\right)_{t}+a_{0}\left(u_{t t}-c_{0} u_{x x}\right)
$$

can be analyzed by integral operator $\mathcal{M}$ (2.8) with conditions (2.10), (2.11) and when the constants $a_{k}, c_{k}$ satisfy (3.8) and (3.11).

\section{Polymeric materials}

Polymeric materials are very flexible like rubber and are easily formed into fibres, thin films, etc. Moreover, the liquid state composed only of polymers (polymer melt) is an important state for industrial uses where polymeric materials are processed into various plastic products such as gaskets, seals, flexible joints, vehicle tires, etc.
Also the durability is a requirement imposed on polymers and polymeric composites and the interest for future development of these materials is increasing more and more.

A large literature treats with polymer physics and as for viscoelastic theories, two models which describe different aspects of the polymer chains, have met with reasonable success: the Rouse model and the reptation model [2-3].

In both cases the memory function $g_{n}(t)$ assumes a form like (1.1).

In fact in the Rouse model function $g_{n}(t)$ is given by:

$$
g_{n}(t)=k_{1} \sum_{h=1}^{n} e^{2 h^{2} \frac{t}{\tau_{1}}}
$$

where the relaxation time $\tau_{1}$ can be calculated by means of experimental results. [3].

When the viscoelastic behaviour is represented by the reptation model, as times increases, the stress function decreases with a relaxation time $\tau_{d}$, and one has:

$$
g_{n}(t)=k \sum \frac{1}{h^{2}} e^{-h^{2} \frac{t}{\tau_{d}}},
$$

where $h$ ranges over odd integer, the constant $k$ depends on the polymer physics and the value of the reptation time $\tau_{d}$ can be fixed according to elasticity experiments [1].

So, if one considers the first two steps in the reptation model, it results: $B_{1}=$ $k, \quad B_{2}=B_{1} / 9, \quad \beta_{1}=1 / \tau_{d}, \quad \beta_{2}=9 \beta_{1}$. Consequently the operator (3.12) is characterized by constants: 


$$
\begin{cases}c_{0}=c^{2} \frac{81}{81+82 k \tau_{d}} & a_{0}=1+\frac{82}{81} k \tau_{d} \\ c_{1}=c^{2} \frac{9}{9+k \tau_{d}} & a_{1}=\frac{10 \tau_{d}^{2}}{9}\left(\frac{1}{\tau_{d}}+\frac{k}{9}\right) \\ c_{2}=c^{2} & a_{2}=\frac{\tau_{d}^{2}}{9}\end{cases}
$$

Analogously, in the Rouse model, beeing $B_{1}=B_{2}=k_{1}, \quad \beta_{1}=2 / \tau_{1}, \quad \beta_{2}=4 \beta_{1}$, one has:

$$
\begin{cases}c_{0}=c^{2} \frac{8}{8+5 k_{1} \tau_{1}} & a_{0}=1+\frac{5 k_{1}}{8} \tau_{1} \\ c_{1}=c^{2} \frac{5}{5+k_{1} \tau_{1}} & a_{1}=\frac{\tau_{1}^{2}}{16}\left(2 k_{1}+\frac{10}{\tau_{1}}\right) \\ c_{2}=c^{2} & a_{2}=\frac{\tau_{1}^{2}}{16}\end{cases}
$$

The wave hierarchies defined by (4.3) or (4.4) are governed by the operator $\mathcal{L}_{1}^{*}$ of the Standard Linear Solid defined, respectively, by:

$$
\begin{aligned}
& \text { (4.5) } \begin{cases}c_{0}=c^{2} \frac{81}{81+82 k \tau_{d}} & c_{1}=c^{2} \\
a_{0}=1+\frac{82}{81} k \tau_{d} & \eta=\frac{81 \tau_{d}}{81+82 k \tau_{d}},\end{cases} \\
& (4.6) \begin{cases}c_{0}=c^{2} \frac{8}{8+5 k_{1} \tau_{1}} & c_{1}=c^{2} \\
a_{0}=1+\frac{5}{8} k_{1} \tau_{1} & \eta=\frac{4 \tau_{1}}{8+5 k_{1} \tau_{1}} .\end{cases}
\end{aligned}
$$

Remark 3.1- As swowed, memory function $g_{n}(t)$ can depend on $h^{2}$. So the approximation to the two first terms appears to be reasonable. However, in many article the model is limited to a single relaxtion time (see. f.i.[15]).

\section{References}

1. Ferry, J. D.: Viscoelastic properties of polymers. New York. Wiles, 1961

2. Doi, M. See, H.: Introduction to Polymer Physics. Oxford: Clarendon Press, 1977

3. Doi, M. Edwards S. F.: The theory of Polymer Dynamics. Oxford: Clarendon Press, 1986

4. Sunyer Balaguer, F. : Approximation of functions by linear combinations of exponentials. Collect. Math. 17, 145-177, (1965).

5. Schwartz, L.: Etude des sommes d'exponentielles. Act. Scient. et Industr. 959, Hermann, Paris (1959).

6. Christensen, R. M.: Theory of Viscolasticity. New York: Academic Press, 1971

7. Whitham, G. B.: Linear and Non linear Waves. New York : John Wiley e Sons, 1974

8. Hunter, S.C.: Mechanics of Continuous media. England: Ellis Horwood, 1976 
9. Haupt, P.: Continuous Mechanics and theory of Materials, Springer 2000

10. Renno, P.: On a Wave Theory for the Operator $\varepsilon \partial_{t}\left(\partial_{t}^{2}-c_{1}^{2} \Delta_{n}\right)+\partial_{t}^{2}-c_{0}^{2} \Delta_{n}$. Ann. Mat. pura e Appl.,136(4) 355389 (1984).

11. Graffi. D. : On the fading memory. Applicable Analysis, 15 (1983) 295311

12. Graffi. D.: Mathematical models and waves in linear viscoestacity. Euromech Colloquium 127 on Waves propagation in viscoelastic media, Pitman Adv. Publ. Comp. Research notes in Math.,52, 1-27 1980

13. Renno, P.: On the Cauchy problem il linear viscoelasticity. Ren. Acc. Naz. Lincei, VIII vol. LXXXV (1983)

14. Renno, P.: On some viscoelastic models. Ren. Acc. Naz. Lincei, VIII vol. LXXV (1983)

15. Ianniruberto,G., Marrucci G.: A simple constiyutive equation for entangled polymers with chain stretch. J. Rheol.45(6) 1305- 1318 (2001) 